Research Article

\title{
Mechanism of Antiwrinkle Finishing of Cotton Fabrics Using Mixed Polycarboxylic Acids
}

\author{
Huaifang Wang $\mathbb{D}^{-}$, Chuanjie Zhang $\mathbb{D}^{D}$, Xudong Chu $\mathbb{D}^{\infty}$, and Ping Zhu \\ College of Textile \& Clothing, Institute of Functional Textiles and Advanced Materials, State Key Laboratory of Bio-fibers and Eco- \\ textiles, Collaborative Innovation Center of Marine Biomass Fibers Materials and Textiles of Shandong Province, Qingdao University, \\ Qingdao 266071, China
}

Correspondence should be addressed to Huaifang Wang; hfwang1980@163.com and Ping Zhu; pzhu99@163.com

Received 28 April 2020; Revised 18 June 2020; Accepted 22 June 2020; Published 10 July 2020

Academic Editor: Nabil Ibrahim

Copyright ( 2020 Huaifang Wang et al. This is an open access article distributed under the Creative Commons Attribution License, which permits unrestricted use, distribution, and reproduction in any medium, provided the original work is properly cited.

The kinetics, activation energy, and mechanism of esterification of cotton by mixed polycarboxylic acids, namely, 1,2,3,4butanetetracarboxylic acid (BTCA) and citric acid (CA), were studied during the curing process of cotton fibers in comparison with the esterification by BTCA alone. The reaction rates of these two polycarboxylic acid finishing systems under different temperatures and the dependency of their reaction rate constants on temperature were studied. Consequently, in the temperature range from $120^{\circ} \mathrm{C}$ to $180^{\circ} \mathrm{C}$, the activation energy of the mixed polycarboxylic acids (BTCA+CA) was obtained as $E_{\mathrm{a}}=40.7 \mathrm{~kJ} / \mathrm{mol}$, which was slightly lower than that for the BTCA finishing system. The reaction occurring between the carboxylic acid group of BTCA and the hydroxyl group of CA was proposed. To confirm and prove the speculation, experiments were conducted to investigate the changes in wrinkle recovery angle and tensile strength retention by changing the concentration of both the single polycarboxylic acid and mixed polycarboxylic acids. The results show that, besides esterification of cotton fibers, citric acid probably functioned as an extender in the curing process, when it was combined with BTCA.

\section{Introduction}

Cotton is a natural cellulose fiber that is widely used in apparel textiles because of its desirable properties like softness, comfort, warmth, and hygroscopic nature. However, the slippage movement of the cellulose molecular chains which were held in position by interchain hydrogen bonds results in wrinkle and crease under external forces during use [1]. This drawback greatly restricts its practical applications. To overcome this problem and endow the cotton fabric with antiwrinkle property, cross-linking of cellulose chains (to inhibit their movement) using appropriate resins or polymers has proved to be effective [2-6]. Formaldehyde-based cross-linking agents were the first group of such finishing agents. Among these, dimethylol dihydroxyethyleneurea (DMDHEU) is one of the most widely used agents for producing intermolecular cross-linking to provide easy care properties to the fiber against deformation [7-9]. Moreover, it also improves the elastic recovery of the fiber after deformation with reactive $N$-methylol groups $[3,10,11]$. However, the fabric treated with compounds containing $N$-methylol group has the problem of releasing formaldehyde, a probable human carcinogen, during manufacturing, wearing, and storage $[1,3]$. Since the 1990 s, extensive efforts $[4,5,12-$ 16] have been made to develop formaldehyde-free, durable, and press finishing agents. Polycarboxylic acids such as 1,2,3,4-butanetetracarboxylic acid (BTCA) and citric acid (CA) displayed interesting potential as cross-linking agents for substrates containing hydroxyl groups, including cellulose, starch, and chitosan [15, 17-20]. Many researchers are investigating the effectiveness of the polycarboxylic acid finishing systems for providing antiwrinkle property $[5,6,21$, $22]$ and the cross-linking mechanism of polycarboxylic acid with the treated cotton fabric [14, 23-25] and are finding appropriate catalysts $[5,24,26,27]$. BTCA has proved to be the most effective cross-linking agent as it can impart excellent antiwrinkle properties to the finished fabric along with good and durable washability and whiteness. However, a higher cost is the most obvious barrier for BTCA that prevents its use at the technical scale. Methods to reduce the cost 
of BTCA finishing technology have been investigated. These include combination with other carboxylic acids such as citric acid (CA) $[28,29]$ because CA is a sustainable chemical, cheap, and eco-friendly. However, it is an inferior wrinkleresistant agent and leaves a yellowing effect on the CAtreated fabrics $[30,31]$.

In our previous work, we investigated the best conditions to optimize the wrinkle recovery properties and strength retention of cotton fabric with the BTCA and BTCA/CA finishing systems using the response surface methodology (RSM) and BBD (Box-Behnken design) [32, 33]. Moreover, we also investigated the influence of operating parameters and coupling effects between them. The results indicated that a combination of BTCA and CA could lead to an improvement in both wrinkle resistance and strength retention properties compared to a single BTCA finishing system under lower curing temperatures. This observation was interesting because CA usually performed well only under relatively harsher conditions $[30,31,34]$. As an attempt to evaluate the reactivity of BTCA/CA, the esterification kinetics of the BTCA/CA finishing system was investigated in comparison with that of the single BTCA system to prove the reactivity and the effectiveness of mixed polycarboxylic acids. The reaction rate constant and activation, as well as the possible mechanism, are also proposed.

\section{Experimental}

2.1. Materials. Desized scoured, bleached, and mercerized pure cotton plain-woven fabrics (weight: $140 \mathrm{~g} / \mathrm{m}^{2}$; warp density: 52.4 ends/cm; weft density: 28.3 picks/cm; and yarn count: 14.75 tex $\times 14.76$ tex) were purchased from Weifang Qirong Textile Co., Ltd., Weifang, China. 1,2,3,4-Butanetetracarboxylic acid (BTCA), sodium hypophosphite (SHP) monohydrate, and hydrochloric acid $(\mathrm{HCl})$ were purchased from China National Pharmaceutical Group Co., Ltd., Shanghai, China. Sodium hydroxide $(\mathrm{NaOH})$ was purchased from Kemiou Chemical Reagent Co., Ltd., Tianjin, China. Silver nitrate $\left(\mathrm{AgNO}_{3}\right)$ and barbital sodium were purchased from Xiya Reagent, Linyi, China. Calcium acetate was purchased from Tianjin Dingshengxin Chemical Co., Ltd., Tianjin, China. Cresol red was purchased from MACKLIN Reagent, Shanghai, China. Thymol blue sodium salt was purchased from Beichen Fangzheng Reagent, Tianjin, China. Barbituric acid was purchased from Shanghai Zhanyun Chemical Co., Ltd., Shanghai, China. Hydrochloric acid $(\mathrm{HCl})$ and barbituric acid were of chemically pure grade, and other reagents were of analytical grade. All reagents were used without any further purification.

2.2. Fabric Treatment. The cotton fabric was first impregnated in an aqueous solution containing both BTCA and sodium hypophosphite (SHP); the $\mathrm{pH}$ of the solution was adjusted to 2.5 using $1 \mathrm{M} \mathrm{NaOH}$. The samples were then padded through two dips and two nips to reach an average wet pickup of about $80 \%$. They were then dried at $80^{\circ} \mathrm{C}$ for $5 \mathrm{~min}$ and cured at a specific temperature for a specific time in a curing oven. In case of investigation of kinetics, the cur- ing process was set at $120^{\circ} \mathrm{C}, 140^{\circ} \mathrm{C}, 160^{\circ} \mathrm{C}$, and $180^{\circ} \mathrm{C}$ for $30 \mathrm{~s}$, $60 \mathrm{~s}, 90 \mathrm{~s}, 120 \mathrm{~s}$, and $150 \mathrm{~s}$.

For investigating the kinetics, the finishing solutions of (i) a BTCA/CA system containing $50 \mathrm{~g} / \mathrm{L}$ of BTCA, $45 \mathrm{~g} / \mathrm{L}$ of $\mathrm{CA}$, and $38 \mathrm{~g} / \mathrm{L}$ of SHP and (ii) a BTCA system containing $73 \mathrm{~g} / \mathrm{L}$ of BTCA and $38 \mathrm{~g} / \mathrm{L}$ of SHP were used to treat the fabrics. These two formulas were the best formula obtained, to optimize the wrinkle resistance and strength retention, in our previous work [32, 33].

In order to calculate the content of free carboxylic acid before the esterification reaction, fabrics were dipped and nipped two times, followed by drying at $80^{\circ} \mathrm{C}$ for $5 \mathrm{~min}$ without exposure to the curing process. Further, the washing process was omitted after curing to calculate the content of esters in the cured fabrics.

\subsection{Characterization of Finished Fabrics}

2.3.1. Wrinkle Recovery Angle (WRA). The WRAs of the treated fabrics were measured according to the American Association of Textile Chemists and Colorists (AATCC) method 66-1990 with an LFY-1 fabric wrinkle recovery tester. Each sample was measured twelve times in both the warp and weft directions to obtain the average value.

2.3.2. Tensile Strength (TS). The tensile strength of the finished fabrics was measured by using a YG026 electronic fabric strength tester according to the ASTM D 5035-90. All measurements were performed in the weft direction to characterize and calculate the strength retention of the treated fabrics.

$$
\text { TS retention }(\%)=\frac{\mathrm{TS}_{i}}{\mathrm{TS}_{0}}
$$

where $\mathrm{TS}_{0}$ and $\mathrm{TS}_{i}$ are the tensile breaking strengths $(N)$ of the fabrics before and after the finishing treatment under various conditions, respectively.

2.3.3. Yellowness Index (YI). The yellowness index of all finished and control cotton fabrics was tested according to ASTM D 1925 (observer: $2^{\circ}$, illuminant: c) [27], which was carried out on a spectrophotometer (X-Rite, Inc., Grand Rapids, MI) under illuminant $c$ using a $2^{\circ}$ standard observer. Four measurements for each fabric sample were taken to obtain the average value.

2.3.4. Carboxyl Content Determination. Calcium acetate titration $[35,36]$ was used for the determination of the carboxyl content of the treated cotton fabrics. This method is based on ion exchange and involves the reaction of the carboxyl groups with calcium acetate, as shown in Scheme 1.

Before titration measurement, the treated fabric was divided into single yarns, kept at room temperature for $24 \mathrm{~h}$, and then dried at $80^{\circ} \mathrm{C}$ to yield a constant weight. About $500 \mathrm{mg}$ of the fabric was placed in a $100 \mathrm{~mL}$ ground-glassstoppered Erlenmeyer flask, and $25 \mathrm{~mL}$ of an aqueous solution of $0.1 \mathrm{M}$ fresh $\left(\mathrm{CH}_{3} \mathrm{COO}\right) \mathrm{Ca}$ was added to it. The blank test was performed separately. After shaking for $2 \mathrm{~h}$ at room temperature, ten drops of the mixed indicators thymol blue 


$$
2(-\mathrm{COOH})+\mathrm{Ca}\left(\mathrm{CH}_{3} \mathrm{COO}\right)_{2} \rightleftharpoons-\mathrm{COO}^{-\mathrm{Ca}+2 \mathrm{CH}_{3} \mathrm{COOH}}
$$

SCHEme 1: Ion exchange between the carboxyl group and calcium acetate.

and cresol red were added into the flask. Titration was carried out using $0.01 \mathrm{M} \mathrm{NaOH}$ solution until the color of the solution changed from yellow to violet.

Carboxylic acid content $\left(c_{(\mathrm{PCA})}\right)$ of the treated fabric, i.e., the nonreacted free carboxylic acid after the curing process, was calculated according to the following equation:

$$
c_{(\mathrm{PCA})}=\frac{V \times c}{W}\left(\frac{\mathrm{mmol}}{\mathrm{g}}\right)
$$

where $V$ is the volume of consumed $\mathrm{NaOH}$ solution, $c$ is the concentration of standard $\mathrm{NaOH}$ solution, and $W$ is the weight of the treated fabric used for titration.

The consumed carboxylic acid was calculated by $c_{0 \text { (PCA) }}$ $-c_{(\mathrm{PCA})}$. Here, $c_{0(\mathrm{PCA})}$ is the carboxylic acid content before curing, and $c_{t(\mathrm{PCA})}$ is the nonreacted carboxylic acid content after being cured for a given period of time.

\section{Results and Discussion}

3.1. Consumption of Polycarboxylic Acids. In order to determine the curing time and temperature range for calculating the kinetics data of esterification, the amount of carboxylic acid consumed in the esterification process during the curing step under different temperatures was measured by the calcium acetate titration as shown in Figures 1 and 2 . The temperatures $120^{\circ} \mathrm{C}, 140^{\circ} \mathrm{C}, 160^{\circ} \mathrm{C}$, and $180^{\circ} \mathrm{C}$ were selected in this investigation. We saw that the consumption of carboxylic acids increased as the curing time prolonged, and a higher amount of carboxylic acids was consumed under higher temperatures for both the finishing systems. This showed that the esterification of carboxylic acids with cellulose was accelerated by temperature. In addition, the consumption increased and was then maintained at a nearly constant level under higher temperatures when the curing time was longer than $120 \mathrm{~s}$. This indicated that the reaction had approached an equilibrium condition.

3.2. Calculation of the Rate Constant of Esterification. According to the reaction mechanism, polycarboxylic acids react with the hydroxyl groups in the cellulose to form an ester linkage and impart wrinkle recovery properties to the treated fabrics. As previously reported, the kinetics of esterification $[37,38]$ between the polycarboxylic acids and the cellulosic hydroxyl groups can be depicted as a pseudofirst-order reaction with respect to the carboxylic acid concentration; the reaction rate $\tau$ was calculated according to the following equation:

$$
\tau=-\frac{d c_{(\mathrm{PCA})}}{d t}=k c_{(\mathrm{PCA})}^{\alpha} c_{(\mathrm{CAT})}^{\beta} c_{(\mathrm{OH})}^{\gamma},
$$

where $k$ is the reaction rate constant, $c_{(\mathrm{PCA})}, c_{(\mathrm{CAT})}$, and $c_{(\mathrm{OH})}$ are the concentrations of the carboxylic acid, catalyst, and hydroxyl groups in cotton cellulose, respectively. $\alpha, \beta$, and $\gamma$ are the orders of the reaction. As the concentration of the catalyst was considered to be constant, the hydroxyl group was considered to be in excess in comparison with the amount of PCA. Hence, it could be assumed to be constant and Equation (3) was transformed into Equation (4) as below:

$$
\tau=-\frac{d c_{(\mathrm{PCA})}}{d t}=k_{1} c_{(\mathrm{PCA})}
$$

By taking the logarithm of both sides of Equation (4), Equation (5) was obtained.

$$
\ln c_{0(\mathrm{PCA})}-\ln c_{t(\mathrm{PCA})}=k_{2} t
$$

where $c_{0(\mathrm{PCA})}$ is the concentration of carboxylic acids before curing, i.e., the carboxylic acid content at the beginning of the esterification, and $c_{t(\mathrm{PCA})}$ is the concentration of carboxylic acids that have not reacted after curing for a specific time. The nonreacted carboxylic acid content in the fabrics, which have undergone curing for different time points, was calculated from the calcium acetate titration method. Thus, we plotted $\ln c_{0 \text { (PCA) }}-\ln c_{t(\mathrm{PCA})}$ against the curing time $(t)$ and obtained the value of the slope that represented the constant rate.

Figures 3 and 4 show the esterification kinetics of carboxylic acid under different temperatures in the BTCA and BTCA-CA finishing systems, respectively. To calculate the esterification rate constant, the regression lines of $\ln c_{0 \text { (PCA) }}$ $-\ln c_{t(\mathrm{PCA})}$ were plotted against the curing time $(t)$. Additionally, the reaction rate constants under different temperatures $\left(120^{\circ} \mathrm{C}, 140^{\circ} \mathrm{C}, 160^{\circ} \mathrm{C}\right.$, and $\left.180^{\circ} \mathrm{C}\right)$ were calculated from which the activation energy was calculated. Each set of data gave a straight line, as shown in Figures 3 and 4, indicating first-order kinetics with respect to the free carboxylic acid content in the treated fabrics. The slopes of the regression lines were the values of the esterification rate constants. The resulted values are listed in Table 1, showing that the rate constants increased with an increase in the curing temperature. This demonstrated that the esterification reaction was faster under higher temperatures.

3.3. Calculation of Activation Energy. Higher temperatures accelerated the process of esterification. The temperature dependence of the rate constant followed the Arrhenius equation, as shown in Equation (6). By taking the logarithm of both sides of Equation (6), a new equation was obtained.

$$
\begin{aligned}
k & =A e^{\left(-E_{\mathrm{a}} / R T\right)}, \\
\ln k & =\ln A-\frac{E_{\mathrm{a}}}{R T},
\end{aligned}
$$

where $A$ is the frequency factor, $E_{\mathrm{a}}$ is the Arrhenius activation energy, $R$ is the gas constant $(8.314 \mathrm{~J} /(\mathrm{mol} \cdot \mathrm{K}))$, and $T$ is the 


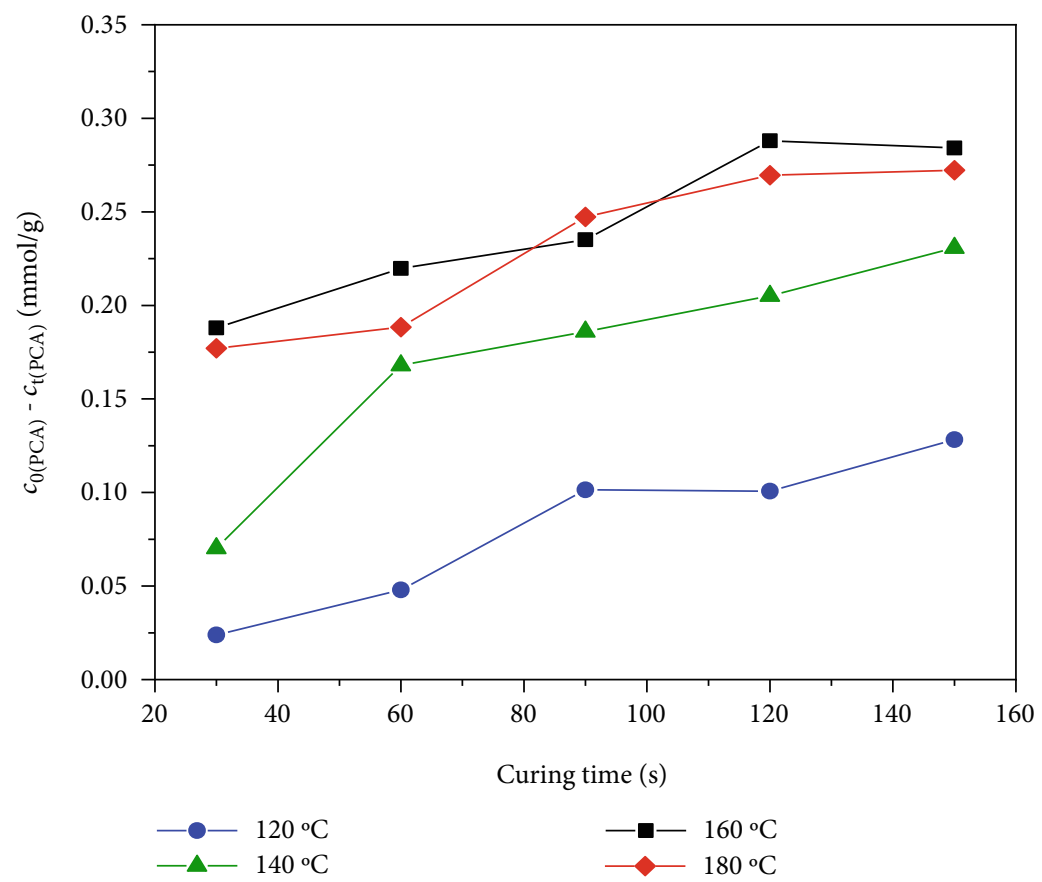

FIgURE 1: Effect of curing time on the consumption of carboxylic acids in the BTCA finishing system.

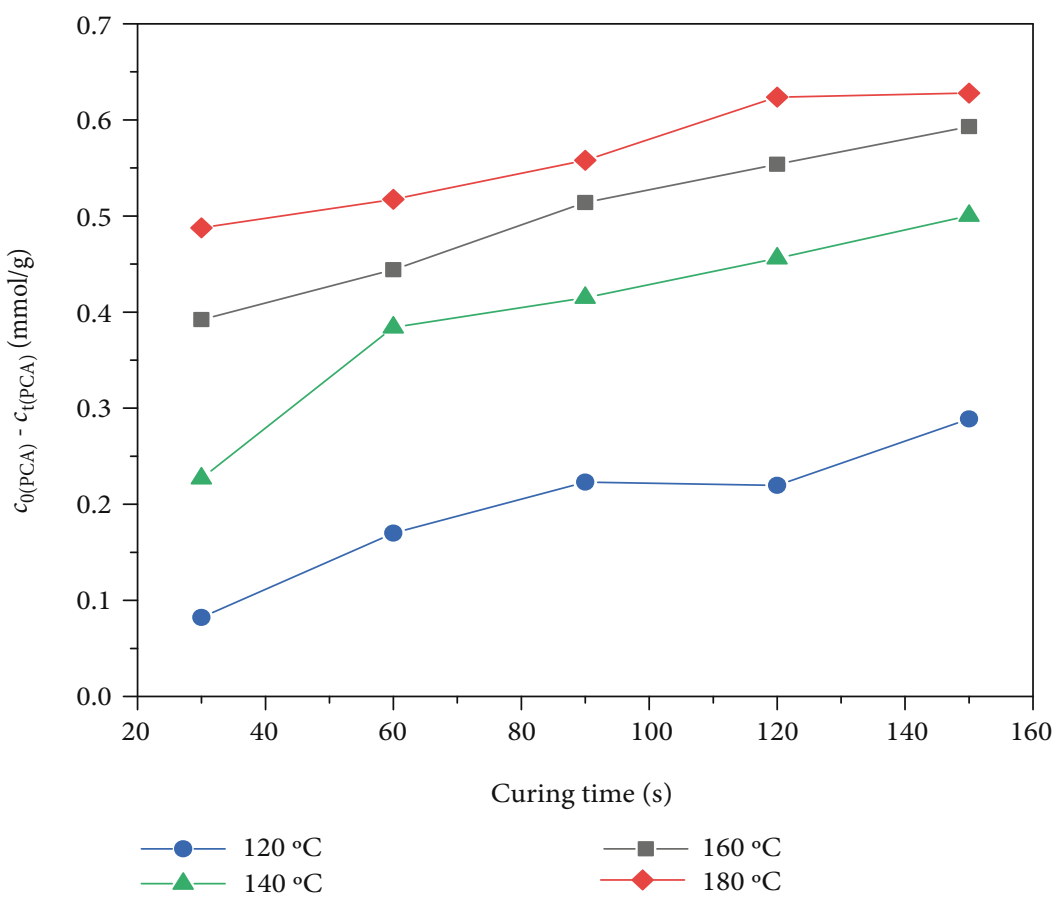

FIGURE 2: Effect of curing time on the consumption of carboxylic acids in the BTCA/CA finishing system.

temperature. According to Equation (7), $E_{\mathrm{a}}$ was calculated by plotting $\ln k$ against $1 / T$. By adding the values of the rate constants from Table 1 and those of the corresponding temperature into Equation (7), the fitting curves of $\ln k$ against $1 / T$ were plotted in Figures 5 and 6.

The slopes and the intercepts of the corresponding regression lines represented $-R / T$ and $\ln A$, respectively.
The overall apparent activation energy using mixed acids of BTCA/CA esterification with hydroxyl groups during the curing process was calculated to be $40.70 \mathrm{~kJ} / \mathrm{mol}$ that was slightly lower than the values for the BTCA esterification $(44.4 \mathrm{~kJ} / \mathrm{mol})$ (Table 1$)$. This suggested that by combining $\mathrm{CA}$ in the BTCA finishing system, the esterification process was easier. This was an interesting observation of a possible 


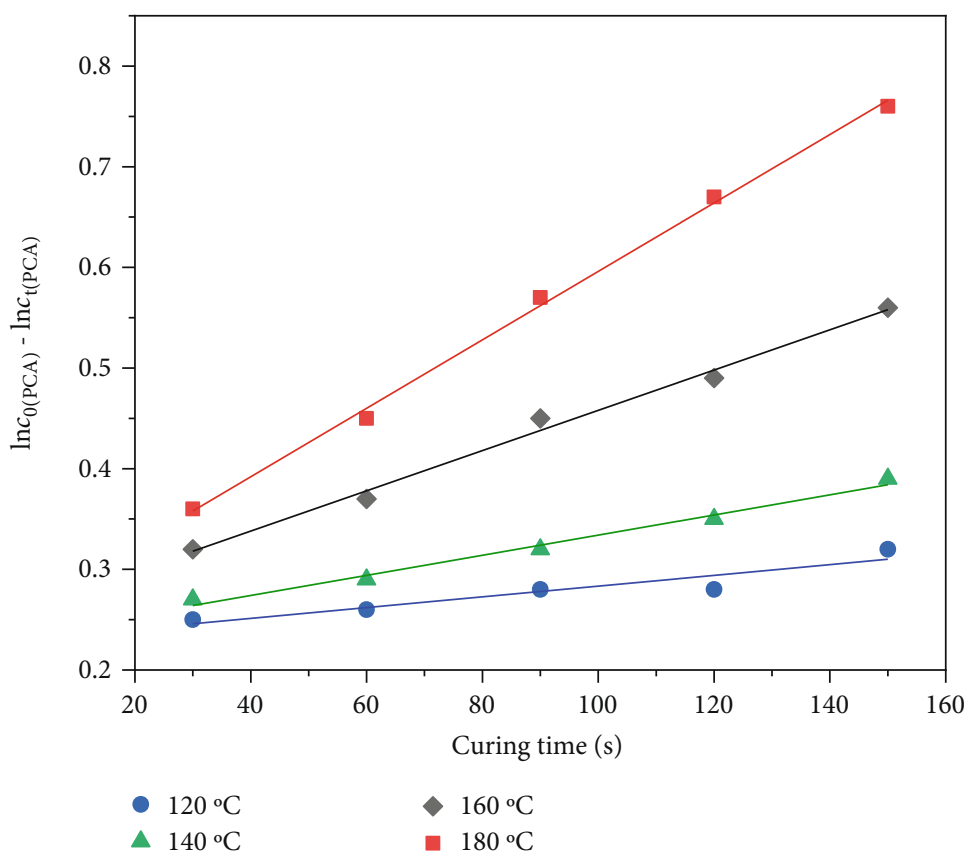

FIGURE 3: Esterification kinetics plot for the BTCA finishing system under various temperatures.

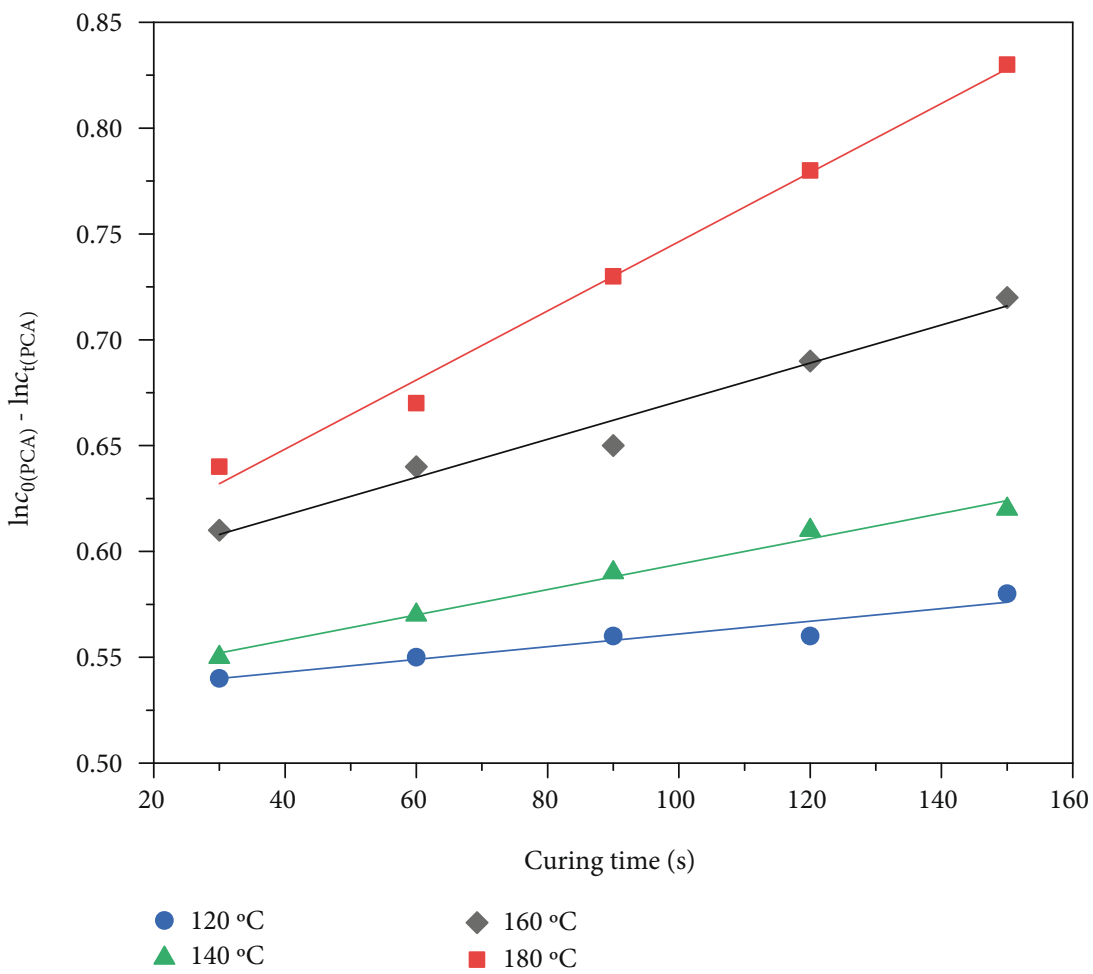

FIGURE 4: Plot of the esterification kinetics for the finishing system combining BTCA and CA under various temperatures.

reaction between BTCA and CA since it was earlier reported that CA had a weaker reactivity than BTCA [37].

\section{Mechanism Analysis}

As shown in the previous report, the $\alpha$-hydroxyl groups in the citric acid molecule hindered its esterification with cellu- lose [39]. Moreover, the presence of other polycarboxylic acids, such as homopolymers and terpolymers, reacted with the hydroxyl group and transformed CA from trifunctional to tetrafunctional molecule. Therefore, the increased reactivity of the BTCA/CA system was interpreted by similar esterification of the carboxylic acids in the BTCA molecule with the hydroxyl groups of citric acid, correspondingly increasing 
TABLE 1: Kinetics data of esterification during the curing process for both the BTCA and BTCA/CA system.

\begin{tabular}{lccccrr}
\hline & & & $k$ & & & $E_{a}(\mathrm{~kJ} / \mathrm{Mol})$ \\
\hline BTCA & $120^{\circ} \mathrm{C}$ & $140^{\circ} \mathrm{C}$ & $160^{\circ} \mathrm{C}$ & $180^{\circ} \mathrm{C}$ & $\mathrm{A}$ & 44.4 \\
BTCA/CA & 0.00057 & 0.001 & 0.002 & 0.00337 & 441.98 \\
\hline
\end{tabular}

$1 / T$

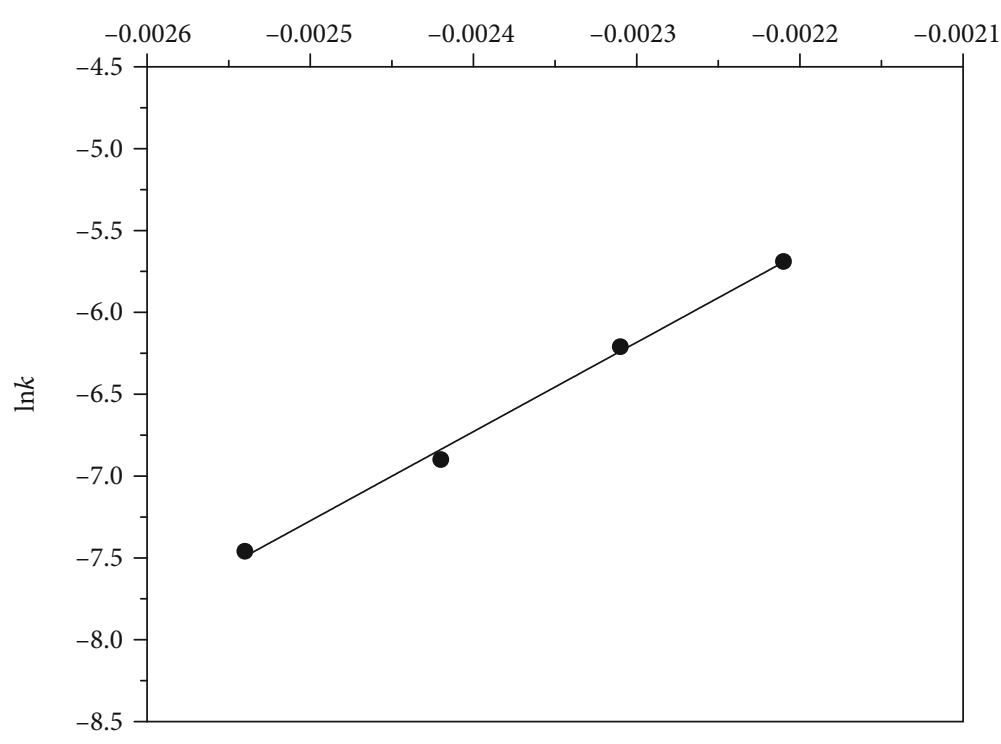

FIgURE 5: Linear fitting plot of $\ln k$ with $1 / T$ of reaction constants of BTCA.

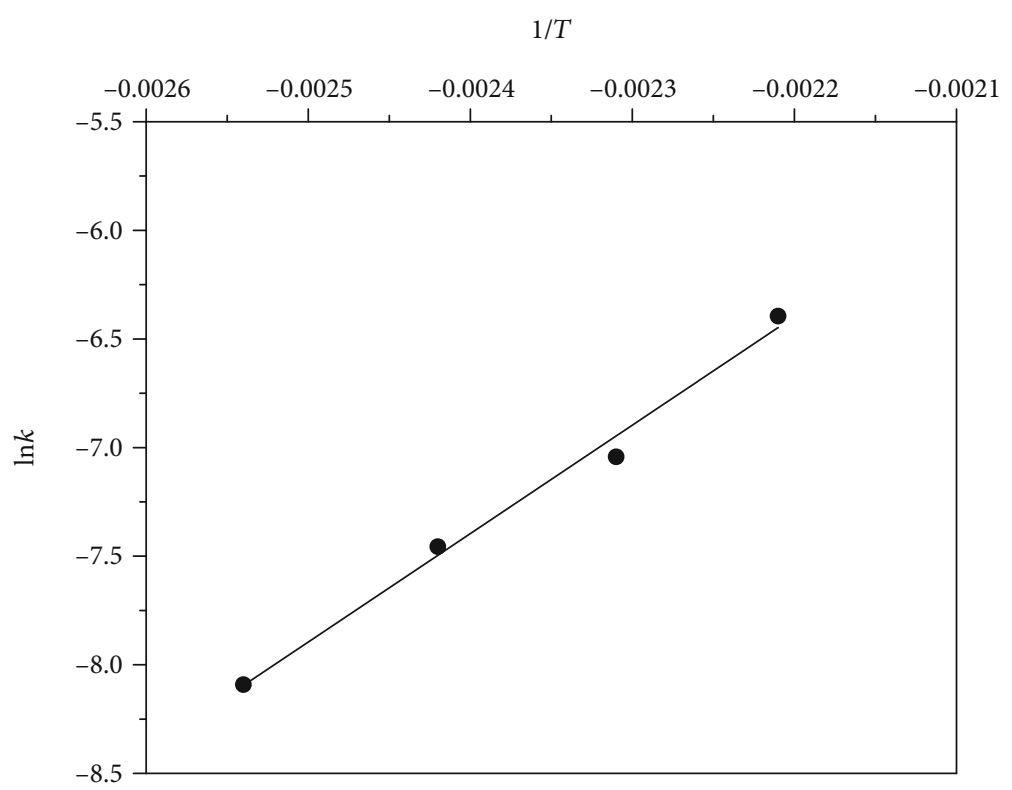

FIGURE 6: Linear fitting plot of $\ln k$ with $1 / T$ of reaction constants of BTCA and CA.

the effectiveness of the BTCA/CA finishing system. With such a possible reaction mechanism in mind, a series of experiments were designed and conducted with the crosslinking of either BTCA or CA or a combination of these two. The fabric samples were impregnated in the following four groups of aqueous solutions: (a) BTCA (from 10 to $90 \mathrm{~g} / \mathrm{L}$ ), (b) CA (from 10 to $90 \mathrm{~g} / \mathrm{L}$ ), (c) BTCA (50 g/L)+CA (from 20 to $70 \mathrm{~g} / \mathrm{L}$ ), and (d) CA $(40 \mathrm{~g} / \mathrm{L})+\mathrm{BTCA}$ (from 10 


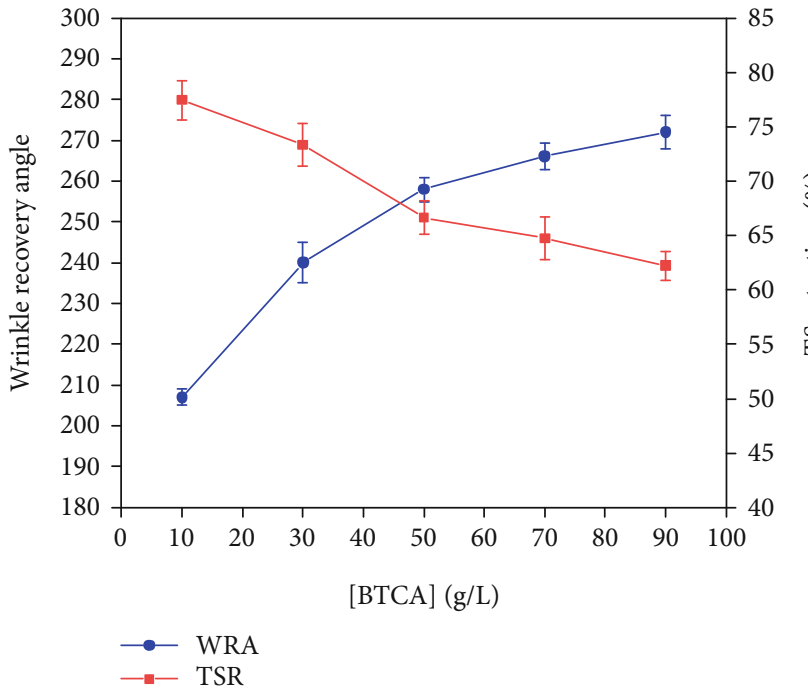

(a)

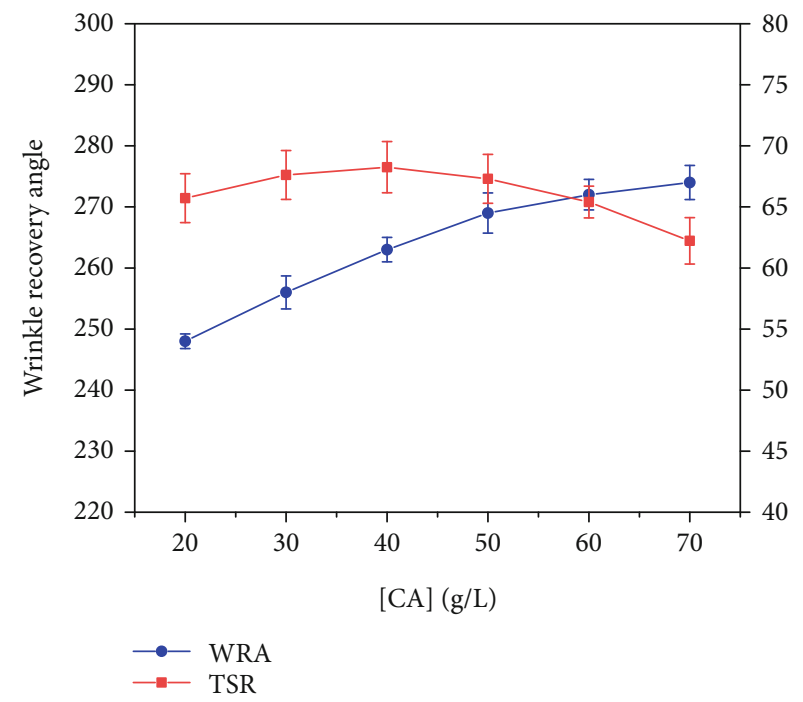

(c)

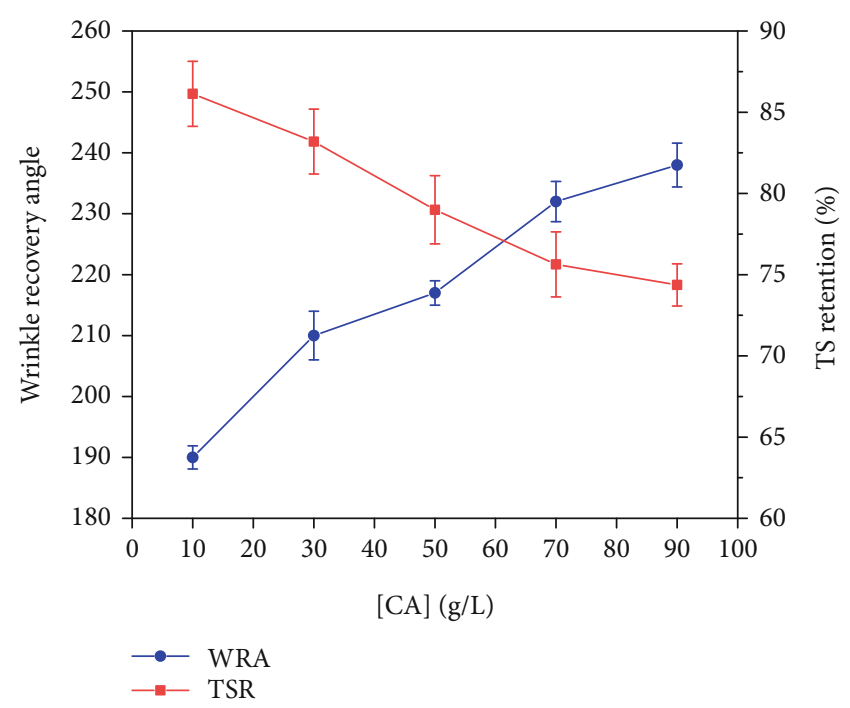

(b)

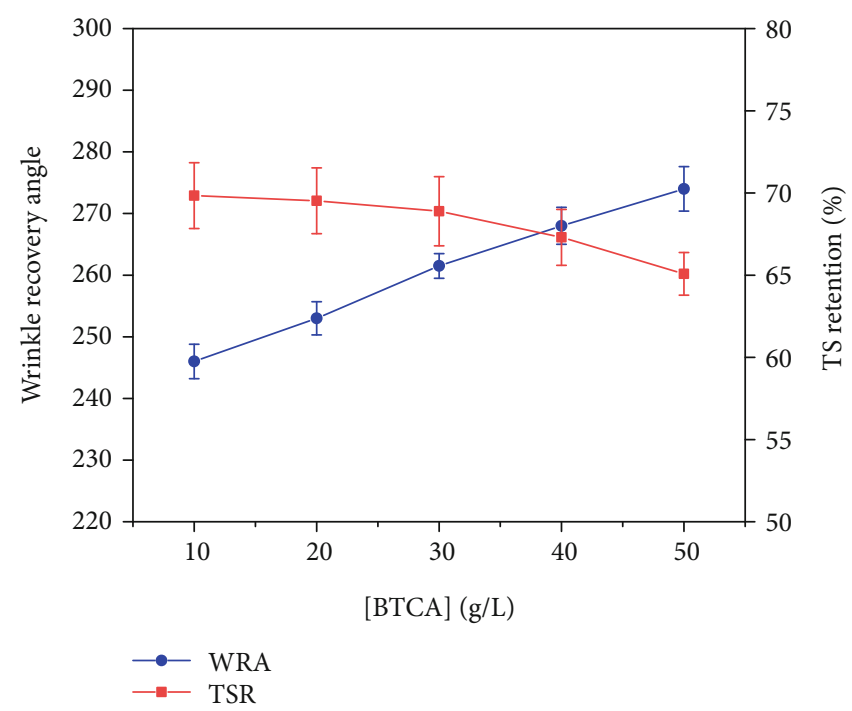

(d)

FIGURE 7: (a) WRA and TS retention of fabrics treated with BTCA. (b) WRA and TS retention of fabrics treated with CA. (c) Effect of changing concentration of CA on fabrics treated with BTCA/CA combination with $50 \mathrm{~g} / \mathrm{L}$ BTCA. (d) Effect of changing concentration of BTCA on fabrics treated with BTCA/CA combination with $40 \mathrm{~g} / \mathrm{L} \mathrm{CA}$.

to $50 \mathrm{~g} / \mathrm{L})$. SHP $(38 \mathrm{~g} / \mathrm{L})$ was the catalyst in all the finishing baths. Subsequently, the impregnated fabrics were dried at $80^{\circ} \mathrm{C}$ for $5 \mathrm{~min}$ and cured at $170^{\circ} \mathrm{C}$ for $90 \mathrm{~s}$.

From Figures 7(a) and 7(b), it was seen that the fabrics treated with either BTCA or CA alone possessed higher WRA and lower tensile strength. It has been demonstrated in earlier reports that the loss of strength in the treated fabrics was attributed to ester cross-linking between polycarboxylic acid and cellulose, besides the process of acidic degradation. However, fabrics treated with the combination of BTCA and CA displayed a different pattern than that shown by fabrics treated with BTCA or CA alone. As seen in Figure 7(c), a slight increase in the strength of the fabric was observed when the concentration of CA increased from 20 to $40 \mathrm{~g} / \mathrm{L}$ in the presence of $50 \mathrm{~g} / \mathrm{L}$ BTCA. This phenomenon could possibly be explained by the esterification of carboxylic acids in BTCA and that of the hydroxyl groups in the CA molecule, which formed longer and flexible cross-linking bridges between the cellulose chains. This bridge formation was very similar to the role of an extender [31]. In addition, this kind of esterification was expected to take place when sufficient BTCA and CA were present as no increase in strength was observed (Figure 7(d)). A possible cross-linking pathway is presented in Scheme 2. The results indicated that there was a coupling effect between BTCA and CA and the fabrics treated with the combination of BTCA $(50 \mathrm{~g} / \mathrm{L})$ and CA (about $40 \mathrm{~g} / \mathrm{L}$ ) displayed a compromised WRA and tensile strength, which was consistent with our previous report [29]. In addition, using mixed polycarboxylic acids also has an advantage of decreasing the yellowness of the treated 

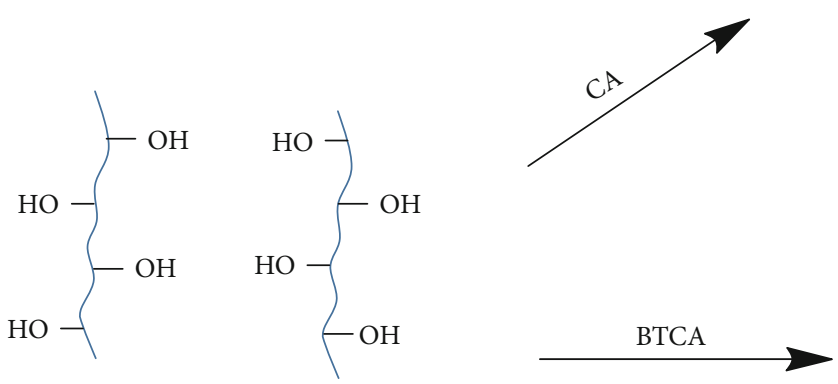<smiles>CC(O)CC(O)CC(CC(C)O)OC(=O)CC(O)(CC(=O)OC(C)CC(O)CC(O)CCOC(=O)CC(O)(CC(=O)O)C(=O)O)C(=O)O</smiles>

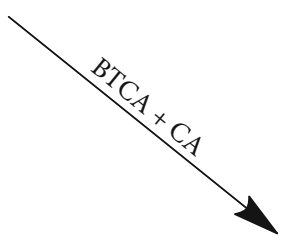<smiles>CC(O)CCC(O)C(O)C(C)OC(=O)CC(C(=O)O)C(CC(=O)OC(C)CC(O)CC(O)C(O)CC(C)OC(=O)CC(C(=O)O)C(CC(=O)O)C(=O)O)C(=O)O</smiles>

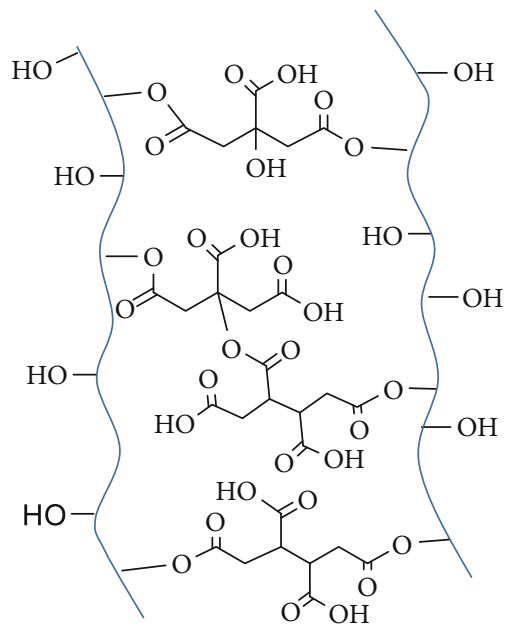

Scheme 2: Possible cross-linking between polycarboxylic acids and cellulose.

fabric when compared to that treated with CA alone, as shown in Table 2, possibly, partially due to the reaction of hydroxyl groups of $\mathrm{CA}$ to the carboxylic acid groups in BTCA and inhibiting formation of unsaturated aconitic acid $[22,31]$.

\section{Conclusion}

The kinetics and activation energy for the esterification of cotton fabric were investigated using different polycarboxylic acids, namely, a combination of BTCA and CA and CA alone. The activation energy of $40.7 \mathrm{~kJ} / \mathrm{mol}$ of BTCA $/ \mathrm{CA}$ was slightly lower than that of BTCA $(44.4 \mathrm{~kJ} / \mathrm{mol})$; this demonstrated a possibly higher reactivity for the BTCA/CA finishing system. A probable reason for this observation was proposed. The proposed mechanism was proved when a change was observed in the pattern of the WRA and tensile strength of the fabrics treated with increasing concentrations of polycarboxylic acids in the BTCA, CA, and BTCA/CA fin-
TABLE 2: Yellowness index of fabrics treated with polycarboxylic acids.

\begin{tabular}{lc}
\hline Samples & YI \\
\hline Control cotton $^{\text {a }}$ & 4.80 \\
BTCA $^{\mathrm{a}}$ & 8.85 \\
$\mathrm{BTCA}^{\mathrm{C}} \mathrm{CA}^{\mathrm{b}}$ & 11.53 \\
$\mathrm{CA}^{\mathrm{c}}$ & 13.26 \\
\hline
\end{tabular}

${ }^{\mathrm{a}}$ Finishing solution: $73 \mathrm{~g} / \mathrm{L} \mathrm{BTCA}$ and $38 \mathrm{~g} / \mathrm{L}$ SHP, cured at $170^{\circ} \mathrm{C}$ for $90 \mathrm{~s}$. ${ }^{\mathrm{b}}$ Finishing solution: $50 \mathrm{~g} / \mathrm{L}$ BTCA, $45 \mathrm{~g} / \mathrm{L} \mathrm{CA}$, and $38 \mathrm{~g} / \mathrm{L}$ SHP, cured at $170^{\circ} \mathrm{C}$ for $90 \mathrm{~s} .{ }^{\mathrm{c}} 100 \mathrm{~g} / \mathrm{L} \mathrm{CA}$ and $38 \mathrm{~g} / \mathrm{L} \mathrm{SHP}$, cured at $170^{\circ} \mathrm{C}$ for $90 \mathrm{~s}$.

ishing systems. The results indicated that CA performed as an extender when sufficient BTCA and CA were present.

\section{Data Availability}

All datasets for this study are included in the manuscript. 


\section{Conflicts of Interest}

The authors declare that they have no competing interests.

\section{References}

[1] V. A. Dehabadi, H.-J. Buschmann, and J. S. Gutmann, "Durable press finishing of cotton fabrics: an overview," Textile Research Journal, vol. 83, no. 18, pp. 1974-1995, 2013.

[2] B. A. K. Andrews, J. M. Simoneaux, and R. J. Harper, "Formaldehyde release and cellulose crosslinking with $\mathrm{N}$-methylol agents: a delicate balance," Angewandte Makromolekulare Chemie, vol. 115, no. 1, pp. 115-129, 1983.

[3] P. Bajaj, "Finishing of textile materials," Journal of Applied Polymer Science, vol. 83, no. 3, pp. 631-659, 2002.

[4] Z.-R. Li, W.-C. Jiang, L.-J. Wang, W.-D. Meng, and F.-L. Qing, "Synthesis and application of novel aqueous anionic polyurethane as a durable press finishing agent of cotton fabrics," Textile Research Journal, vol. 77, no. 4, pp. 227-232, 2016.

[5] C. M. Welch, "Tetracarboxylic acids as formaldehyde-free durable press finishing agents," Textile Research Journal, vol. 58, no. 8, pp. 480-486, 1988.

[6] C. Q. Yang and X. Wang, "Formation of cyclic anhydride intermediates and esterification of cotton cellulose by multifunctional carboxylic acids_an infrared spectroscopy study," Textile Research Journal, vol. 66, no. 9, pp. 595-603, 2016.

[7] N. A. Ibrahim, A. Bayazeed, R. Refai, and A. Hebeish, "Some basics of easy-care cotton finishing. Part IX: semicarbazide $\mathrm{HCl}$ - a new catalyst," American Dyestuff Reporter, vol. 75, no. 5 , p. 13, 1986.

[8] N. A. Ibrahim, R. Refai, and A. Hebeish, Basics of easy-care cotton finishing. XI. Improved performance with modified magnesium chloride catalysts, American Dyestuff Reporter, 1986.

[9] N. A. Ibrahim, B. M. Eid, E. A. El-Aziz, T. M. Abou Elmaaty, and S. M. Ramadan, "Multifunctional cellulose-containing fabrics using modified finishing formulations," RSC Advances, vol. 7, no. 53, pp. 33219-33230, 2017.

[10] H. Petersen and P. S. Pai, "Reagents for low-formaldehyde finishing of textiles," Textile Research Journal, vol. 51, no. 4, pp. 282-302, 2016.

[11] H. Petersen, "Recent developments in the chemistry of crosslinking Agents," Textile Research Journal, vol. 40, no. 4, pp. 335-344, 2016.

[12] C. M. Welch, "Glyoxal as a formaldehyde-free durable press reagent for mild curing applications," Textile Research Journal, vol. 53, no. 3, pp. 181-186, 2016.

[13] M. Hashem, P. Hauser, and B. Smith, "Wrinkle recovery for cellulosic fabric by means of ionic crosslinking," Textile Research Journal, vol. 73, no. 9, pp. 762-766, 2016.

[14] C. Q. Yang, "FT-IR spectroscopy study of the ester crosslinking mechanism of cotton cellulose," Textile Research Journal, vol. 61, no. 8, pp. 433-440, 2016.

[15] M. M. G. Fouda, A. el Shafei, S. Sharaf, and A. Hebeish, "Microwave curing for producing cotton fabrics with easy care and antibacterial properties," Carbohydrate Polymers, vol. 77, no. 3, pp. 651-655, 2009.

[16] N. A. Ibrahim, B. M. Eid, and S. M. Sharaf, "Functional finishes for cotton-based textiles: current situation and future trends," in Textiles and Clothing, M. Shabbir, Ed., pp. 131-190, John Wiley \& Sons, Inc. and Scrivener Publishing LLC USA, 2019.
[17] H. M. Fahmy and M. M. G. Fouda, "Crosslinking of alginic acid/chitosan matrices using polycarboxylic acids and their utilization for sodium diclofenac release," Carbohydrate Polymers, vol. 73, no. 4, pp. 606-611, 2008.

[18] M. M. G. Fouda and H. M. Fahmy, "Multifunctional finish and cotton cellulose fabric," Carbohydrate Polymers, vol. 86, no. 2, pp. 625-629, 2011.

[19] N. A. Ibrahim, B. M. Eid, E. A. el-Aziz, T. M. A. Elmaaty, and S. M. Ramadan, "Loading of chitosan - nano metal oxide hybrids onto cotton/polyester fabrics to impart permanent and effective multifunctions," International Journal of Biological Macromolecules, vol. 105, Part 1, pp. 769-776, 2017.

[20] L. Shen, H. Xu, L. Kong, and Y. Yang, "Non-toxic crosslinking of starch using polycarboxylic acids: kinetic study and quantitative correlation of mechanical properties and crosslinking degrees," Journal of Polymers and the Environment, vol. 23, no. 4, pp. 588-594, 2015.

[21] A. Hou and G. Sun, "Multifunctional finishing of cotton fabrics with 3,3',4,4'-benzophenone tetracarboxylic dianhydride: reaction mechanism," Carbohydrate Polymers, vol. 95, no. 2, pp. 768-772, 2013.

[22] N. A. Ibrahim, M. H. Abo-Shosha, E. I. Elnagdy, and M. A. Gaffar, "Eco-friendly durable press finishing of cellulose-containing fabrics," Journal of Applied Polymer Science, vol. 84, no. 12, pp. 2243-2253, 2002.

[23] C. Q. Yang, "Infrared spectroscopy studies of the cyclic anhydride as the intermediate for the ester crosslinking of cotton cellulose by polycarboxylic acids. I. Identification of the cyclic anhydride intermediate," Journal of Polymer Science, vol. 31, no. 5, pp. 1187-1193, 1993.

[24] B. Ji, H. Qi, K. Yan, and G. Sun, "Catalytic actions of alkaline salts in reactions between 1,2,3,4-butanetetracarboxylic acid and cellulose: I Anhydride formation," Cellulose, vol. 23, no. 1, pp. 259-267, 2016.

[25] B. Ji, P. Tang, K. Yan, and G. Sun, "Catalytic actions of alkaline salts in reactions between 1,2,3,4-butanetetracarboxylic acid and cellulose: II. Esterification," Carbohydr Polym, vol. 132, pp. 228-236, 2015.

[26] C. Zhao and G. Sun, "Catalytic actions of sodium salts in direct esterification of 3,3'4,4'-benzophenone tetracarboxylic acid with cellulose," Industrial \& Engineering Chemistry Research, vol. 54, no. 43, pp. 10553-10559, 2015.

[27] A. Nazari, M. Montazer, A. Rashidi, M. Yazdanshenas, and M. Anary-Abbasinejad, "Nano TiO2 photo-catalyst and sodium hypophosphite for cross-linking cotton with poly carboxylic acids under UV and high temperature," Applied Catalysis A: General, vol. 371, no. 1-2, pp. 10-16, 2009.

[28] C. Schramm, S. B. Vukusic, and D. Katovic, "Non-formaldehyde durable press finishing of dyed fabrics_ evaluation of cotton-bound polycarboxylic acids," Coloration Technology, vol. 118, no. 5, pp. 244-249, 2002.

[29] X. Chu, H. Wang, P. Zhu, and S. Sui, "Opitmization of durable press finishing process of cotton fabric with combination of BTCA and CA by response surface mothodology," Basic Sciences Journal of Textile Universities, vol. 32, no. 2, pp. 1-9, 2019.

[30] P. Tang, B. Ji, and G. Sun, "Whiteness improvement of citric acid crosslinked cotton fabrics: $\mathrm{H} 2 \mathrm{O} 2$ bleaching under alkaline condition," Carbohydrate Polymers, vol. 147, pp. 139-145, 2016.

[31] W. Yao, B. Wang, T. Ye, and Y. Yang, "Durable press finishing of cotton fabrics with citric acid: enhancement of whiteness and 
wrinkle recovery by polyol extenders," Industrial \& Engineering Chemistry Research, vol. 52, no. 46, pp. 16118-16127, 2013.

[32] X. Chu, H. Wang, P. Zhu, and S. Sui, "Optimization of DP finish with mixed polycarboxylic acid by reponse surface methodology," Dyeing and Printing, vol. 21, pp. 16-21, 2018.

[33] X. Chu, H. Wang, S. Sui, and P. Zhu, "Modeling and opimization of durable press finishing of cotton fabric with BTCA by response surface methodology," AATCC Journal of Research, vol. 7, no. 4, pp. 37-44, 2020.

[34] C. Q. Yang, L. Xu, S. Li, and Y. Jiang, "Nonformaldehyde durable press finishing of cotton fabrics by combining citric acid with polymers of maleic acid," Textile Research Journal, vol. 68, no. 6, pp. 457-464, 2016.

[35] H. N. S. Schafer, "Carboxyl groups and ion exchange in lowrank coals," Fuel, vol. 49, no. 2, pp. 197-213, 1970.

[36] C. Y. Jeong, C. W. Park, J. G. Kim, and S. K. Lim, "Carboxylic content of humic acid determined by modeling, calcium acetate, and precipitation methods," Soil Science Society of America Journal, vol. 71, no. 1, pp. 86-94, 2007.

[37] C. Schramm, B. Rinderer, and O. Bobleter, "Kinetic data for the crosslinking reaction of polycarboxylic acids with cellulose," Journal of the Society of Dyers and Colourists, vol. 113, no. 12 , pp. 346-349, 1997.

[38] H. Qi, Formaldehyde-free anti-wrinkle finishing on cotton fabrics by polycarboxlic acids, Donghua University, 2016.

[39] C. Q. Yang, X. Wang, and I.-S. Kang, "Ester crosslinking of cotton fabric by polymeric carboxylic acids and citric acid," Textile Research Journal, vol. 67, no. 5, pp. 334-342, 1997. 